\title{
Anmerkungen zur Entwicklung der Rechtsprechung des Gerichts- hofes auf dem Gebiet des Sozialrechts
}

\author{
Thomas von DANWITZ
}

\section{Einführung}

Der Überblick, den mein akademischer Kollege Becker über die Entwicklung der Rechtsprechung des Europäischen Gerichtshofes zum Sozialrecht gegeben hat, macht jeden Versuch einer ergänzenden Berichterstattung überflüssig und ermöglicht es vielmehr, die mir zugewiesene Aufgabe eines Koreferates zu nutzen, um Anmerkungen zu drei grundlegenden Fragestellungen der Entwicklung des Europäischen Sozialrechts in der Rechtsprechung des Gerichtshofes zu machen. Eine erste Bemerkung soll einem Grunderfordernis des staatlichen Rechts der Sozialleistungserbringung gewidmet sein: Dem Wohnsitzerfordernis, welches die Gewährungspraxis in den Mitgliedstaaten weitgehend prägt. Eine zweite Bemerkung gilt der Rechtsprechung zur Unionsbürgerschaft und der Entfaltung ihrer sozialen Dimension, die den Unionsbürgern unter Umständen Zugang zu den Sozialleistungssystemen der Mitgliedstaaten verschaffen kann. Abschließend möchte ich auf eine Systemfrage des gesamten Sozialversicherungsrechts zu sprechen kommen, welche die Möglichkeiten und Grenzen einer Anwendung der Wettbewerbsvorschriften des Vertrages auf die Aufgabenerfüllung durch die Sozialversicherungsträger betrifft.

\section{Das sozialrechtliche Wohnsitzerfordernis in der Rechtsprechung des Gerichtshofes}

Nach der weiten Auslegung, die der Begriff der „,sozialen Vergünstigungen“ in Art. 7 Abs. 2 der VO Nr. 1612/68 seit jeher in der Rechtsprechung des Gerichtshofes ${ }^{1}$ zur Arbeitnehmerfreizügigkeit erfahren hat, sind unter „sozialen Vergünstigungen“ alle Leistungen zu verstehen, die inländischen Arbeitnehmern wegen ihrer objektiven Arbeitnehmereigenschaft oder einfach wegen ihres Wohnortes im Inland allgemein gewährt werden ${ }^{2}$. Auf dieser Grundlage erfassen die Vorgaben des Gemeinschaftsrechts

$1 E u G H$, Slg. 1975, 1085, Rn. 12 f. - Christini; Slg. 1992, I-1071, Rn. 26 - Bernini; Slg. 1996, I2617, Rn. 17 - O’Flynn; Slg. 1997, 6689, Rn. 45 - Meints.

2 Ebenda, Urteil Meints, Rn. 39; EuGH, Slg. 1998, I-2691, Rn. 25 - Maria Martinez Sala. 
weite Bereiche der Gewährung mitgliedstaatlicher Sozialleistungen. So verbietet der Grundsatz der Gleichbehandlung, der ebenso aus der Arbeitnehmerfreizügigkeit des Art. 39 EG wie aus Art. 7 der VO Nr. 1612/68 folgt, nicht nur offene Diskriminierungen auf Grund der Staatsangehörigkeit, sondern er erfasst in ständiger Rechtsprechung auch alle verdeckten Formen der Diskriminierung, die durch die Anwendung anderer Unterscheidungsmerkmale tatsächlich zu dem gleichen Ergebnis führen ${ }^{3}$. Daher ist eine Vorschrift des nationalen Rechts, die nicht objektiv gerechtfertigt ist und in einem angemessenen Verhältnis zum verfolgten Zweck steht, als mittelbare Diskriminierung anzusehen, wenn sie ihrem Wesen nach geeignet ist, sich auf die sog. Wanderarbeitnehmer eher als auf inländische Arbeitnehmer auszuwirken und deshalb die Gefahr besteht, Wanderarbeitnehmer zu benachteiligen ${ }^{4}$.

Vor dem Hintergrund dieser Rechtsprechung ist die Voraussetzung der Ansässigkeit bzw. des Wohnsitzes im Inland für die Gewährung sozialer Leistungen als mittelbare Diskriminierung anzusehen. Sie muss daher objektiv gerechtfertigt werden können und in einem angemessenen Verhältnis zu dem mit der Leistungsgewährung verfolgten Ziel stehen. Das wird bei zunehmender Mobilität, nicht zuletzt bei Inländern problematisch, die eine soziale Leistung ins Ausland mitnehmen ${ }^{5}$. Nachdem es in der Vergangenheit in zwei Fällen nicht gelungen war, den Gerichtshof von der Erforderlichkeit eines Inlandswohnsitzes zu überzeugen ${ }^{6}$, hat das $B S o z G$ mit den Rechtssachen Hartmann \& Geven dem Gerichtshof die Frage nach dem Wohnsitzerfordernis des deutschen Erziehungsgeldgesetzes zur Beantwortung vorgelegt. Wie Generalanwalt Geelhoed in seinen Schlussanträgen hervorgehoben hat, kann kein Zweifel bestehen, dass die Mitgliedstaaten mit vollem Recht eine Politik zur Förderung der Geburtenrate verfolgen dürfen. Ebenso unzweifelhaft besteht keine gemeinschaftsrechtliche Verpflichtung, zur demographischen Entwicklung anderer Mitgliedstaaten beizutragen ${ }^{7}$. Daher wird der Gerichtshof vor allem zu entscheiden haben, ob und unter welchen Umständen das Wohnsitzerfordernis ein tatsächlich notwendiges Kriterium darstellt, um die vom Zweck des Erziehungsgeldes vorausgesetzte enge Verbindung zu der Gesellschaft des jeweiligen Mitgliedstaates zu gewährleisten oder ob es darüber hinausgeht. Insofern kann sich der Gerichtshof an seiner Rechtsprechung orientieren, wonach die Auszahlung von Beihilfen für Arbeitsuchende von einer tatsächlichen Verbindung mit dem Arbeitsmarkt des betreffenden Mitgliedstaates abhängig gemacht werden darf ${ }^{8}$.

3 Urteile Meints, Rn. 44 u. O’Flynn, Rn. 17.

4 Ebenda, Rn. 45 des Urteils Meints und Rn. 20 des Urteils O’Flynn.

5 Vgl. für die Kinder von Wanderarbeitnehmern, die eine Leistung mitnehmen, EuGH, Slg. 1990, I4185 - di Leo.

6 EuGH, Slg. 1993, I-817, Rn. 12, 15, 34 - Kommission/Luxemburg; Urteil Meints, Rn. 48. Hinzuweisen ist insoweit namentlich auf die Verfahrensbeteiligung der Mitgliedstaaten in der Rechtssache Bidar, Slg. 2005, I-2119.

7 SchlA Geelhoed in Hartmann, C-212/05, anhängig.

8 EuGH, Slg. 2004, I-2703, Rn. 66 - Collins; Slg. 2006, I-6947, Rn. 41 bis 48 - De Cuyper. Vgl. für Unterhaltsbeihilfen, allerdings im Rahmen von Art. 18 EG, Urteil Bidar, Rn. 57-61. 


\section{Die Entfaltung einer sozialen Leistungsdimension der Unionsbürger- schaft}

Damit komme ich zur Frage der Entfaltung einer sozialen Leistungsdimension der Unionsbürgerschaft in der Rechtsprechung des Gerichtshofes ${ }^{9}$. Mag diese Entwicklung in verschiedenen Mitgliedstaaten eine nachhaltige Befürwortung erfahren haben ${ }^{10}$, ist sie in Deutschland ungewöhnlich heftig kritisiert ${ }^{11}$, ja sogar als „Ende rationaler Jurisprudenz" 12 gegeißelt worden. So berechtigt und geboten eine sachliche Kritik der Rechtswissenschaft gerade gegenüber einem Höchstgericht erscheint, das außerhalb der dogmatischen Traditionslinien der nach wie vor mitgliedstaatlich verwurzelten Kultur rechtswissenschaftlicher Analyse und Diskussion steht, so sehr sollte es darum gehen, eine rationale, der rechtswissenschaftlichen Methodenlehre verpflichtete Rechtsfindung in der Rechtsprechung des Gerichtshofes zu stärken. Jenseits dessen ist bemerkenswert, dass die höchst unterschiedlichen Reaktionen auf die Rechtsprechung des Gerichtshofes zur Unionsbürgerschaft zu einem Großteil die ebenso verschiedenartigen Erwartungshaltungen widerspiegeln, welche die Einfügung der Unionsbürgerschaft in den Vertrag und namentlich die Aufnahme der allgemeinen Freizügigkeit in Art. 18 EG ausgelöst hat ${ }^{13}$. Vor diesem Entstehungshintergrund war wohl kaum zu erwarten, dass der Unionsbürgerschaft in der Rechtsprechung des Gerichtshofes eine lediglich deklaratorische und redaktionelle Bedeutung zuerkannt werden würde ${ }^{14}$.

Indes ist die Dogmatik der Unionsbürgerschaft nach wie vor wenig gefestigt ${ }^{15}$. So reicht die Palette der Ansichten über ihre Rechtsnatur vom Grundrecht 16 über die Grundfreiheit 17 bis zum grundlegenden Status ${ }^{18}$ der Angehörigen der Mitgliedstaaten. In der Sache selbst ist der Hinweis allerdings fraglos zutreffend, dass die Unionsbürgerschaft der Gemeinschaft keine Zuständigkeit für die Vergabe von Sozialleistungen verleiht und zudem unter dem Vorbehalt steht, der sich aus Art. 18 Abs. 1 EG selbst ergibt $^{19}$. Dennoch bleibt darauf hinzuweisen, dass das Fehlen einer Sachkompetenz zu

9 Urteile Martinez Sala und Bidar; EuGH, Slg. 2003, I-11613 - Garcia Avello; Slg. 2001, I-6193 Grzelcyk; Slg. 2002, I-6191 - d'Hoop; Slg. 2002, I-7091 - Baumbast.

10 Vgl. nur SchlA Cosmas Slg. 1999, I-6207, ab Rn. 37 - Wijsenbeek.

11 Siehe Jürgen Schwarze, Grundrechtsschutz durch den EuGH, NJW 2005, S. 3459 (3463 f.).

12 So Kay Hailbronner, Die Unionsbürgerschaft und das Ende rationaler Jurisprudenz durch den EuGH ?, NJW 2004, S. 2185 ff.

13 SchlA Cosmas in Wijsenbeek, Rn. 37 einerseits, Rn. 38 bis 42 andererseits.

14 Darauf macht Meinhard Hilf in seiner Kommentierung von 2001 aufmerksam, siehe dens., in: Grabitz-Hilf (Hrsg.), Art. 18 EGV Rn. 21.

15 SchlA Geelhoed in Baumbast, Rn. 63.

16 So SchlA Cosmas in Wijsenbeek, Rn. 95; Siegfried Magiera in Streinz (Hrsg.), EUV/EGV, Art. 18 EGV, Rn. 10.

17 So wohl in Urteil Garcia Avello, Rn. 24.

18 SchlA Geelhoed in Bidar, Rn. 28.

19 So Schwarze, (Fn.), NJW 2005, S. 3459 (3463 f.). 
Gunsten der Gemeinschaft nach ständiger Rechtsprechung die Mitgliedstaaten nicht von der Beachtung der Anforderungen zu entbinden vermag, die sich aus den Grundfreiheiten bzw. Grundrechten ergeben ${ }^{20}$. Zudem berücksichtigt die Kritik an der Rechtsprechung vielleicht zu wenig, dass es sich in den Rechtssachen Grzelczyk und Bidar ${ }^{21}$ um sehr eigentümliche, vom Einzelfall geprägte Sachverhaltskonstellationen handelte, die nicht ohne weiteres einer Verallgemeinerung zugänglich sind. Schließlich sollte stärker beachtet werden, dass der Gerichtshof in dem Urteil Grzelczyk ausdrücklich betont hat, die öffentlichen Finanzen des Aufnahmemitgliedstaates dürften nach der einschlägigen Richtlinie nicht „über Gebühr“ belastet werden. Daraus hat er e contrario geschlossen, dass die Richtlinie eine gewisse finanzielle Solidarität der Angehörigen des Aufnahmemitgliedstaates mit den Angehörigen anderer Mitgliedstaaten anerkennt ${ }^{22}$. In dem Urteil Bidar hat der Gerichtshof weitergehend ausgeführt, dass es jedem Mitgliedstaat freistehe, darauf zu achten, dass die Gewährung von Beihilfen zur Deckung des Unterhalts von Studenten aus anderen Mitgliedstaaten nicht zu einer übermäßigen Belastung erwächst, die Auswirkungen auf das gesamte Niveau der Beihilfen haben könnte, die dieser Staat gewähren kann ${ }^{23}$. Daher sei es namentlich legitim, dass ein Mitgliedstaat solche Beihilfen nur Studenten gewährt, die nachgewiesen haben, dass sie sich bis zu einem gewissen Grad in die Gesellschaft dieses Staates integriert haben ${ }^{24}$. Einen entsprechenden Verweis auf die finanzielle Stabilität findet sich auch in der ständigen Rechtsprechung zu den Auswirkungen der Grundfreiheiten auf die Sozialversicherungssysteme der Mitgliedstaaten 25 .

Dem Gerichtshof ist also durchaus bewusst, dass die Unionsbürgerschaft den Bürgern zwar genuin gemeinschaftsrechtlich begründete Rechte verleiht, es aber die Mitgliedstaaten sind, welche die Kosten für die Verwirklichung dieser Rechte unter Umständen zu tragen haben. Der Gerichtshof wird in der anhängigen Rechtssache Morgan und Bucher erneut Gelegenheit haben, sich mit dieser ,anormalen“ Situation auseinanderzusetzen - wie mein Kollege Arestis sie bezeichnet hat ${ }^{26}$. In dieser Rechtssache stellt sich die Frage nach der eingeschränkten Förderungsfähigkeit von Auslandsstudien nach dem BAFöG erstmals allgemein ${ }^{27}$. Überdies wirft dieser Fall die Frage nach der leistungsrechtlichen Dimension der Unionsbürgerschaft in voller Breite auf, denn die Vorlage des VG Aachen beruht gleichsam auf der Prämisse, dass eine im Vergleich zu

20 Urteil Garcia Avello, Rn. 24. Zur Voraussetzung einer engen Verbindung mit der Gesellschaft des betreffenden Mitgliedstaates bereits oben bei Fn. 8 .

21 Nachweise in Fn. 9.

22 Urteil Grzelczyk, Rn. 44.

23 Urteil Bidar, Rn. 56.

24 Urteil Bidar, Rn. 57.

$25 E u G H$, Slg 1998, I-1931, Rn. 41- Kohll; Slg. 2001, I-5473, Rn. 72 f., 97 - Smits u. Peerbooms; Slg. 2003, I-4509, Rn. 73 - Müller-Fauré.

26 So George Arestis, Towards an EU citizenship?, Annual lecture at the Manchester Jean Monnet Center of Excellence and the Manchester European Research Institute, 16.3.2007, p. $10 \mathrm{f}$.

27 Vgl. für die Kinder von Wanderarbeitnehmern, Urteil di Leo. 
Inlandsstudien eingeschränkte Förderung von Auslandsstudien eine Beschränkung der Freizügigkeit nach Art. 18 EG darstelle, also im Grundsatz von einem unionsbürgerschaftlichen Anspruch auf Gewährung von Leistungen für Inlands- und Auslandsstudien unter gleichen Voraussetzungen auszugehen sei, der nur unter bestimmten Voraussetzungen beschränkt werden könne. Damit dürfte sich die Diskussion um die Bedeutung des Wohnsitzerfordernisses zu einem Gutteil in den Bereich der Unionsbürgerschaft verlagern.

\section{Zur Anwendung des Wettbewerbsrechts auf die Sozialversicherungssys- teme}

Eine für das gesamte System der Sozialversicherung und namentlich für die gesetzliche Krankenversicherung grundlegende Frage betrifft die Anwendbarkeit der Wettbewerbsvorschriften. Für bestimmte Einrichtungen der gesetzlichen Kranken- und Rentenversicherung hat der Gerichtshof entschieden, dass sie einen rein sozialen Zweck verfolgen und keine wirtschaftliche Tätigkeit ausüben, so dass sie dem Unternehmensbegriff des europäischen Wettbewerbsrechts nicht unterfallen.

Dies ist der Fall, wenn die Einrichtungen keine Möglichkeit haben, auf die Höhe der Beiträge, die Verwendung der Mittel und die Bestimmung des Leistungsumfangs Einfluss zu nehmen, da ihre auf dem Grundsatz der nationalen Solidarität beruhende Tätigkeit ohne Gewinnerzielungsabsicht ausgeübt wird und die Leistungen von Gesetzes wegen unabhängig von der Höhe der Beiträge erbracht werden ${ }^{28}$. Die gleiche Beurteilung trifft für Einrichtungen zu, die kraft Gesetzes mit der Versicherung gegen Arbeitsunfälle und Berufskrankheiten betraut sind, weil Leistungen und Beiträge letztlich vom Staat festgelegt werden ${ }^{29}$. Diese für die italienische Unfallversicherungsanstalt in der Rechtssache Cisal vom Gerichtshof vorgenommene Beurteilung ist allgemein formuliert und daher auf vergleichbare Systeme übertragbar. Es ist daher prima vista einleuchtend, warum das $B S o z G$ für die Beurteilung der gesetzlichen Unfallversicherung Deutschlands von einem acte eclairé ausgegangen ist und mithin von einer Vorlage an den Gerichtshof abgesehen hat ${ }^{30}$. In der Rechtssache FENIN hat der Gerichtshof überdies entschieden, dass die Beurteilung der Einkaufstätigkeit von Einrichtungen der sozialen Sicherheit nicht von dem Verwendungszweck zu trennen ist und der wirtschaftliche bzw. nichtwirtschaftliche Charakter der späteren Verwendung der erworbenen Erzeugnisse zwangsläufig den Charakter der Einkaufstätigkeit bestimmt ${ }^{31}$.

28 EuGH, Slg. 1993, I-637, Rn. 5 und 18 - Poucet \& Pistre; Slg. 2004, I-2493, Rn. 47 - AOKBundesverband.

29 EuGH, Slg. 2002, I-691, Rn. 43 bis 46 - Cisal.

$30 B S G$, Urteil vom 9.5.2006, B 2 U 34/05 R, Rn. 15 ff.

$31 E u G H$, Slg. 2006, I-6295, Rn. 26 - FENIN. 
Demgegenüber hat der Gerichtshof Einrichtungen, die zwar gesetzliche Systeme der sozialen Sicherheit verwalten, aber die Höhe der Beiträge und der Leistungen selbst bestimmen und nach dem Kapitalisierungsprinzip arbeiten, als eine wirtschaftliche Tätigkeit im Wettbewerb mit privaten Anbietern angesehen, die den Wettbewerbsvorschriften des Vertrages unterfallen ${ }^{32}$. Vor dem Hintergrund dieser Differenzierung ist es verständlich, dass die in Deutschland im letzten Jahr verabschiedete Reform der gesetzlichen Krankenversicherung, die zu einer Verstärkung von Wettbewerbselementen führt, einen Anlass bietet, um die Frage nach der Anwendung der gemeinschaftlichen Wettbewerbsvorschriften erneut zu diskutieren. Deshalb möchte ich betonen, dass selbst eine unterstellte Anwendbarkeit der Wettbewerbsvorschriften keineswegs einen gänzlichen Umbruch der Systemgrundlagen bedingen würde oder gar müsste.

Denn der Gerichtshof hat eine spezifische Ausnahme von den Wettbewerbsvorschriften nach Art. 86 Abs. 2 EG nicht erst als gerechtfertigt angesehen, wenn das finanzielle Gleichgewicht oder das wirtschaftliche Überleben der mit einer Dienstleistung von allgemeinem wirtschaftlichen Interesse betrauten Einrichtung bedroht ist. Vielmehr genügt es nach der ständigen Rechtsprechung, dass besondere Befugnisse erforderlich sind, um die Erfüllung der im Allgemeinen wirtschaftlichen Interesse liegenden Aufgabenstellung zu wirtschaftlich annehmbaren Bedingungen zu ermöglichen ${ }^{33}$. Besonders hervorzuheben ist insoweit, dass der Gerichtshof den Mitgliedstaaten angesichts der komplexen Natur einer Bewertung der Auswirkungen solcher Befugnisse auf die betroffenen Leistungssysteme und ihr finanzielles Gleichgewicht ausdrücklich einen weiten Ermessensspielraum konzediert hat ${ }^{34}$. Seine gemeinschaftsrechtliche Überprüfung der Erforderlichkeit solcher Sonderrechte hat er spiegelbildlich auf offensichtliche Beurteilungsfehler beschränkt ${ }^{35}$. Diese Perspektive zeigt, dass selbst eine Anwendbarkeit der Wettbewerbsvorschriften, sollte sie zunehmend wettbewerblich ausgerichtete Systeme der sozialen Sicherheit tatsächlich erfassen, kein notorisch zu eng geratenes Korsett bildet, welches der Wahrnehmung sozialer Zielsetzungen entgegenstehen muss. Vielmehr bezeugt die Rechtsprechung zu Art. 86 Abs. 2 EG, dass sich der Gerichtshof dem berechtigten Anliegen der Mitgliedstaaten gegenüber stets aufgeschlossen gezeigt hat, wenn diese eine ausgleichende Durchsetzung von solidarisch-sozialstaatlichen Zielsetzungen mit Instrumenten des Wettbewerbs für geboten erachten, bspw. um eine verbesserte Kostenkontrolle gewährleisten zu können.

$32 E u G H$, Slg. 1995, I-4013, Rn. 17 und 22 - FFSA; Slg. 1999, I-5751, Rn. 81, 84 - Albany.

$33 E u G H$, Slg. 1993, I-2533, Rn. 14-16 - Corbeau; Slg. 1997, 5699, Rn. 37-65 - Stromhandelsmonopole; Urteil Albany, Rn. 107; Slg. 2001, I-4109, Rn. 54 f. - TNT Traco; Slg. 2001, I-8089 - Ambulanz Glöckner.

34 Urteil Albany, Rn. 119.

35 Urteil Albany, Rn. 120. 


\title{
Die Rechtsprechung des EuGH als bestimmender Faktor für die weitere Entwicklung der Koordinierung der Sozialversicherung innerhalb der Gemeinschaft
}

\author{
Sebastião Nóbrega PIZARRO
}

\section{Einführung}

1. Auch wenn es absurd ist, sollte man sich einmal vorstellen, dass es im EG-Vertrag keinen Artikel 177 (heute 234) gäbe, der die Zuständigkeit des Gerichtshofes bei Auslegung und Bewertung der Gültigkeit von Gemeinschaftsrecht regelt - und ich beschränke mich nur auf die an sich schon komplexen Vorschriften, die die Gesetzgebung zur sozialen Sicherheit der 27 Mitgliedstaaten regelt, welche ihrerseits vielfältig und komplex sind und oft unterschiedlichen Konzepten folgen. Dann ginge man zumindest das ernste Risiko ein, dass diese nicht einheitlich ausgelegt würden ${ }^{1}$.

Da dies im Widerspruch zum Integrationsprozess selbst steht, wäre eine noch schwerwiegendere Folge, dass schließlich der soziale Schutz in Frage gestellt würde, der den Unionsbürgern geschuldet wird, die ihr Recht auf Freizügigkeit im EU-Raum wahrgenommen haben oder wahrnehmen.

Nehmen wir auf der anderen Seite die Darlegung der Gründe, z.B. in der Verordnung Nr. 647/2005 zur Änderung der Verordnung 1408/71, bezüglich der Anwendung der Systeme der sozialen Sicherheit auf Arbeitnehmer und Selbständige sowie deren Familienangehörige, die innerhalb der Gemeinschaft $\mathrm{zu}$ und abwandern. Es mussten bestimmte Änderungen an der Verordnung vorgenommen werden, um namentlich die jüngste Rechtsprechung des Gerichtshofes der Europäischen Gemeinschaft zu berücksichtigen und dazu mussten Schlussfolgerungen aus verschiedenen Urteilen gezogen werden. Übrigens ist es auf diesem Gebiet so, dass die meisten Verordnungen mit Reformcharakter identische Erwägungen haben, während dies bei Verordnungen zur Abänderung früherer Verordnungen nur selten der Fall ist.

1 Der Art. 234 des Vertrages hatte ja gerade zum Ziel, einen Mechanismus zu schaffen, der die einheitliche Auslegung der gemeinschaftlichen Rechtsnormen sicherstellt, also einen Mechanismus, der wie man sehen wird, im Bereich der gemeinschaftlichen Koordinierungsregeln für soziale Sicherheit reichlich benutzt wurde. Anders ist es bei anderen multilateralen Koordinierungsregeln, z.B. werden beim Europäischen Abkommen über Soziale Sicherheit des Europarates von 1972 die Probleme der Auslegung durch Verhandlungen der streitenden Parteien gelöst oder, wenn die Differenzen für alle Vertragsparteien von Interesse sind und auf anderem Wege nicht beigelegt werden können, durch das Ministerkomitee, indem im Prinzip vom Präsidenten des Europäischen Gerichtshofes für Menschenrechte ein Schiedsrichter ernannt wird (vgl. Art. 71 des Europäischen Abkommens). 